\title{
Assessment of Left Ventricular Dyssynchrony During Development of Heart Failure by a Novel Program Using ECG-Gated Myocardial Perfusion SPECT
}

\author{
Naoto Takahashi, MD; Akira Yamamoto, MD*; Shingo Tezuka, MD; Masahiro Ishikawa, MD; \\ Junko Abe, MD; Kenichi Amitani, MD; Tomoyoshi Yamaguchi, MD; Naomi Kawaguchi, MD; \\ Takahiro Uchida, MD; Shinichiro Iwahara, MD; Kazuo Munakata, MD
}

\begin{abstract}
Background A novel program, "cardioGRAF", has been developed to analyze regional left ventricular (LV) systolic/diastolic function and dyssynchrony, so the present study aimed to use it confirm the presence of LV dyssynchrony, and to correlate LV function and dyssynchrony with plasma B-type natriuretic peptide (BNP) levels during the early to advanced stages of heart failure (HF).

Methods and Results Fourteen control subjects (G-C) and 50 patients (New York Heart Association functional class I: G-1, 21 patients; class II: G-2, 15 patients; and class III: G-3, 14 patients) were examined by ECG-gated myocardial perfusion single-photon emission computed tomography, using the new index of dyssynchrony, maximal difference (MD), which is the difference between the earliest and latest temporal parameters among 17 segments. First-third filling rate (FR) and the MD of time to peak FR revealing diastolic dyssynchrony were significantly different between G-C subjects and G-1 patients. Ejection fraction, peak ejection rate, peak FR, MD of time to end-systole, and MD of time to peak ejection rate were significantly correlated with plasma BNP levels.

Conclusion Diastolic dyssynchrony was demonstrated even in the early stage of HF, but, although not correlated with the plasma BNP level, systolic dyssynchrony might affect it. (Circ J 2008; 72: 370-377)
\end{abstract}

Key Words: B-type natriuretic peptide; Dyssynchrony; Heart failure; Single-photon emission computed tomography

$\Delta$ pproximately $50 \%$ of heart failure (HF) patients present with a normal left ventricular ejection fraction (LVEF) 1,2 and are generally referred to as having HF caused by left ventricular (LV) diastolic dysfunction, which is diagnosed as diastolic HF (DHF). DHF carries a substantial risk of repeated hospital admissions and a high mortality rate, similar to systolic HF (SHF), but it has been argued that DHF should be treated differently4 Although previous investigators have reported diagnostic criteria for LV diastolic dysfunction, a gold standard has not yet been established.

Recently, it was proven that not only LV systolic/diastolic dysfunction, but also LV dyssynchrony, plays an important role in the advanced stage of HF. In the low LVEF stage, LV wall motion synchronicity between the septum and the free wall is impaired, which leads to LV systolic dysfunction, shortening of the period of LV diastolic inflow and an increase in mitral regurgitation? Wang et al reported that LV systolic/diastolic dyssynchrony occurs in both DHF and SHF, but they evaluated LV dyssynchrony with approxi-

(Received June 17, 2007; revised manuscript received October 18, 2007; accepted October 23, 2007)

Department of Internal Medicine, Nippon Medical School, Musashikosugi Hospital, Kawasaki, *Department of Radiology, Nippon Medical School, Tama-nagayama Hospital, Tokyo, Japan

Mailing address: Naoto Takahashi, MD, Department of Internal Medicine, Nippon Medical School, Musashikosugi Hospital, 1-396 Kosugi-machi, Nakahara-ku, Kawasaki 211-8533, Japan. E-mail address: yukkie@nms.ac.jp

All rights are reserved to the Japanese Circulation Society. For permissions, please e-mail: cj@j-circ.or.jp mately only 4 LV segments using tissue Doppler imaging6

Nuclear cardiology is a modality that evaluates cardiac function precisely. In particular, ECG-gated myocardial perfusion single-photon emission computed tomography (GMPS) enables simultaneous evaluation of various LV functions, with temporal and spatial resolution?,-10 and we have developed a novel program, "cardioGRAF", to analyze regional LV systolic/diastolic function and dyssynchrony.

On the other hand, it has been argued that the level of plasma B-type natriuretic peptide (BNP) is not only a strong marker of LV dysfunction, but also an accurate predictor of morbidity and mortality in patients with HF. Furthermore, elevation of plasma BNP, and the presence of diastolic relaxation or filling abnormalities might help reinforce the diagnosis of LV diastolic dysfunction in patients with preserved LVEF. However, although plasma BNP adds modest discriminatory value in differentiating DHF from SHF, its major role is still the separation of patients with $\mathrm{HF}$ from those without HF!1,12 Therefore, in the present study, we used cardioGRAF to confirm the presence of LV dyssynchrony, and to correlate LV function and dyssynchrony with plasma BNP levels during the early to advanced stages of HF.

\section{Methods}

\section{Patient Population}

Sixty-four patients who were examined by GMPS from November 2000 to April 2006 were studied (Table 1). Of them, the control group (G-C) comprised 14 patients who complained of chest pain or minor ECG abnormality, but 
Table 1 Patients' Characteristics

\begin{tabular}{lccccc}
\hline \hline & Control & & \multicolumn{3}{c}{ Heart failure } \\
\cline { 2 - 2 } \cline { 5 - 6 } & $G-C(n=14)$ & & $G-1(n=21)$ & $G-2(n=15)$ & $G-3(n=14)$ \\
\hline Age & $58 \pm 13$ & & $63 \pm 13$ & $68 \pm 11$ & $66 \pm 14$ \\
Male & 3 & & 15 & 7 & 11 \\
Typical angina & 0 & & 7 & 3 & 0 \\
History of MI & 0 & & 3 & 3 & 0 \\
Arrhythmia & 0 & & 5 & 1 & 5 \\
Cardiomyopathy & 0 & & 2 & 2 & 5 \\
Valvular disease & 0 & & 0 & 3 & 0 \\
Other cardiac disease & $66 \pm 11$ & & $70 \pm 13$ & $70 \pm 13$ & $71 \pm 11$ \\
Resting HR & & & &
\end{tabular}

Data are expressed as means $\pm S D$.

$M I$, myocardial infarction; $H R$, heart rate.

showed no signs of HF that required treatment. The HF group comprised 50 patients with a clinical history of HF based on the Framingham criteria and diagnosed in the past year (33 males, 17 females; $66 \pm 13$ years) $!^{13}$ The etiology of HF was angina pectoris in 10 , myocardial infarction in 10 , arrhythmia in 6 , cardiomyopathy in 11 , valvular disease in 10 , and other causes in 3 . These patients were classified into 3 groups using the functional classification of the New York Heart Association (NYHA): NYHA I (G-1, 21 patients); NYHA II (G-2, 15 patients); NYHA III (G-3, 14 patients). Plasma BNP levels were also measured in 36 patients with HF using a commercially available specific immunoradiometric assay kit (SRL BNP Kit). Exclusion criteria included permanent atrial fibrillation and unstable ventricular rhythm.

\section{Data Acquisition and Analysis}

A single dose of technetium-99m sestamibi (600MBq) was administered intravenously at rest and GMPS was initiated 30-60 min later. Data acquisition was performed using a parallel dual-head gamma camera (Hitachi RW 2600i, Hitachi Corporation, Tokyo, Japan) equipped with low-energy, high-resolution collimators. A total of 64 projections (step and shoot mode, 40-50 beats/projection) were obtained over a $360^{\circ}$ circular orbit. Acquisitions were gated for 16 frames/cardiac cycle. The GMPS images were reconstructed using filtered back projection. Neither attenuation nor scatter correction was used.

\section{Preprocessing for cardioGRAF Analysis}

To determine the position of the inner LV edge, we used a program called "pFAST" (perfusion and function assessment by means of gated SPECT) ${ }^{8}$ version 2.4 , which was developed at Sapporo Medical University, Hokkaido, Japan. Acquired images were preprocessed by p-FAST, as previously described. ${ }^{10}$ The results thus obtained were saved as pFAST data files for future processing with the cardioGRAF program.

\section{cardioGRAF Analysis}

The pFAST data files contained regional volume data in terms of the number of voxels in each phase. Based on the American Heart Association Scientific Statement, we used a 17-segment LV model and divided the regional volume data into 17 segments using a previously described procedure ${ }^{10,11}$ (Fig 1). Global and regional time-volume curves were generated from the data by Fourier curve-fitting analysis with 2 harmonics; the first derivative curve was simultaneously created from the time-volume curve as shown in Figs 2 and 3. The global and regional parameters were obtained from the time-volume curves and the first derivative curves as shown in Fig 2.

\section{Global LV Functional and Temporal Parameters}

The selected functional and temporal parameters were as follows. The LVEF and peak ejection rate (PER), which represents global LV systolic function, and peak filling rate (PFR) and first-third filling rate (1/3FR), which represent global LV diastolic function, were obtained in each case. PFR was defined as the maximum FR during the whole diastolic period in this study. The regional time to end systole (TES) and time to peak ejection (TPE), which represent systolic temporal parameters, the time from end systole to peak filling during the whole diastolic period (TPF1) and the time from 0 to peak filling during the whole diastolic period (TPF2), which represent diastolic temporal parameters in each LV segment, were obtained using the new program.

\section{Index of Heterogeneity or Synchrony of LV Wall Motion}

We propose a new index of dyssynchrony as a measure of LV wall motion heterogeneity; namely, the maximal difference (MD), which is the time lag between the earliest and latest temporal parameters in all 17 segments in each case (Fig 3). MD-TES and MD-TPE represent regional LV systolic dyssynchrony, and MD-TPF1 and MD-TPF2 represent regional LV diastolic dyssynchrony. The severity of LV systolic/diastolic dyssynchrony is expressed as the MD value.

\section{Statistical Analysis}

Values are expressed as means \pm SD. Comparisons of functional indices and MDs among G-C, G-1, G-2 and G-3 were made using Scheffe's F test. The correlations between functional indices, dyssynchrony indices and plasma BNP level were examined using Pearson's correlation coefficient test. A probability of less than 5\% was considered to represent a statistically significant difference.

\section{Results}

\section{Reproducibility of cardioGRAF Data}

The interobserver reproducibility of manual adjustment with the pFAST program was evaluated by 3 operators, and intra-observer reproducibility was evaluated by repeating the process 3 times in 20 consecutive patients. The reproducibility was assessed by the coefficient of variation error. Respective inter-/intra-observer reproducibilities were 4.8\%/3.2\% for TES, $8.0 \% / 7.9 \%$ for TPE, $12.9 \% / 11.0 \%$ for TPF1, and $4.8 \% / 4.4 \%$ for TPF2. 


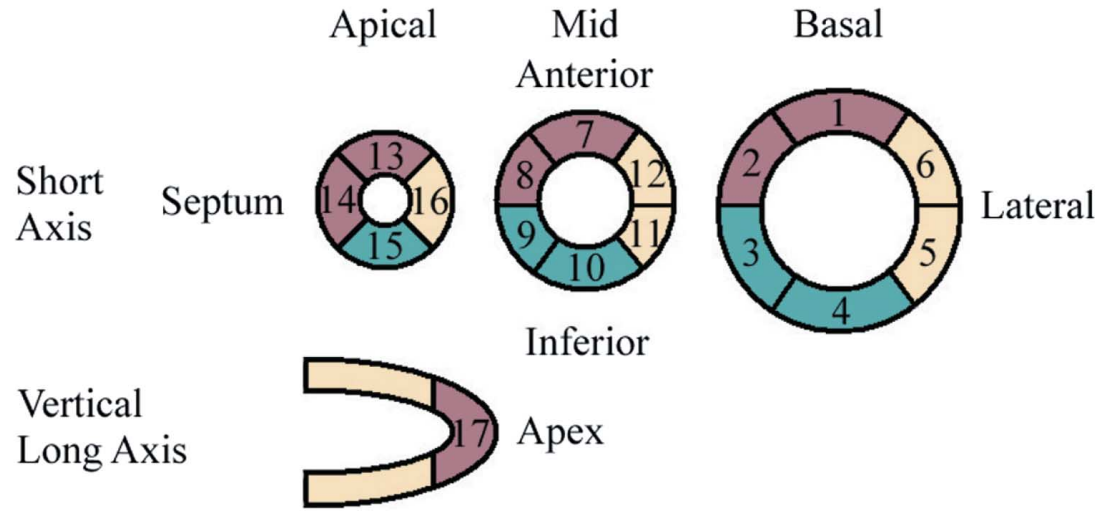

Fig 1. The 17 left ventricular segment model endorsed by the American Heart Association, The American Society of Nuclear Cardiology and many other professional societies.

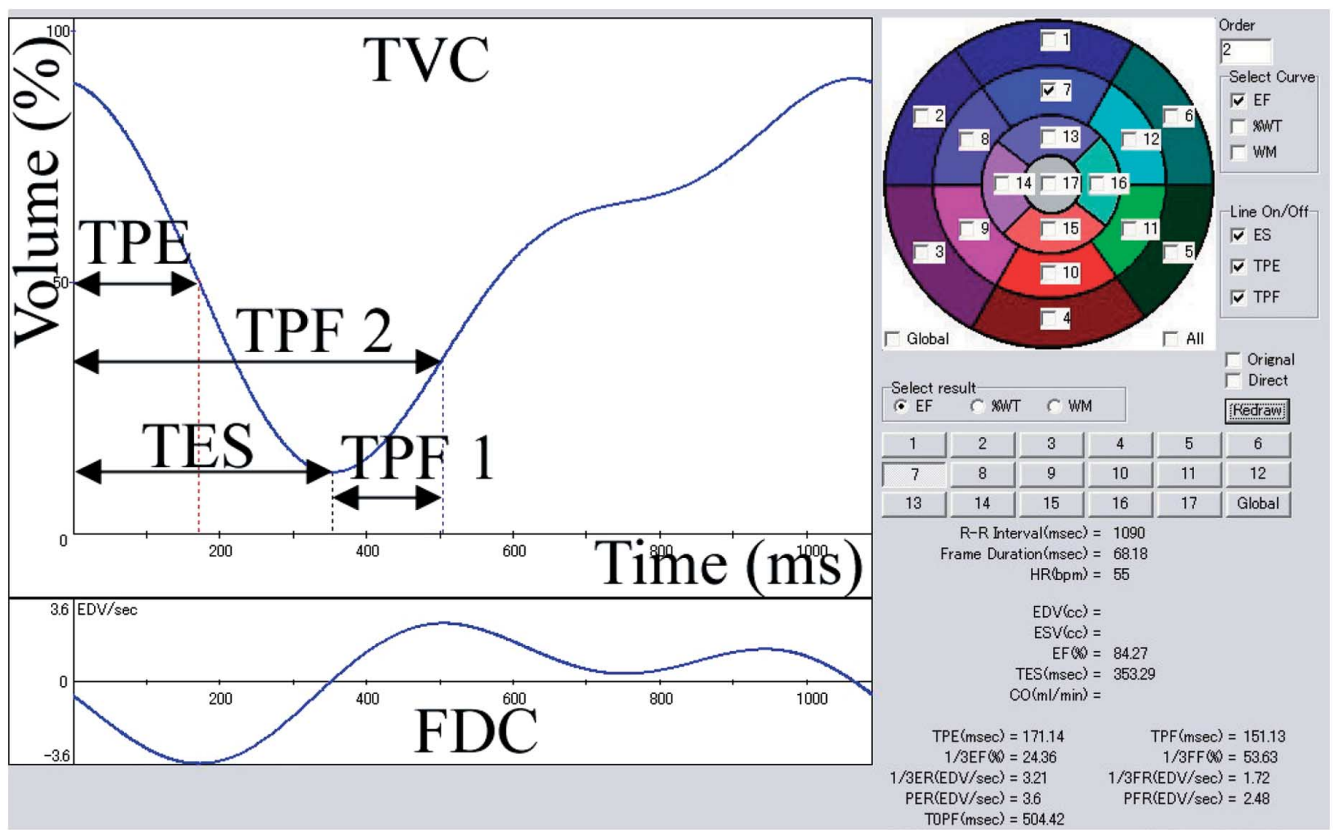

Fig 2. The main window of "cardioGRAF". Original graph, TVC of the global left ventricle and FDC obtained by GMPS at rest (Left). The color polar map shows a circle divided into 17 segments; functional values are illustrated within the segments (Right). TVC, time-volume curve; FDC, first derivative curve; TES, time to end systole (ms); TPE, time to peak ejection (ms); TPF1, time to peak filling (ms); TPF2, time 0 to peak filling (ms).

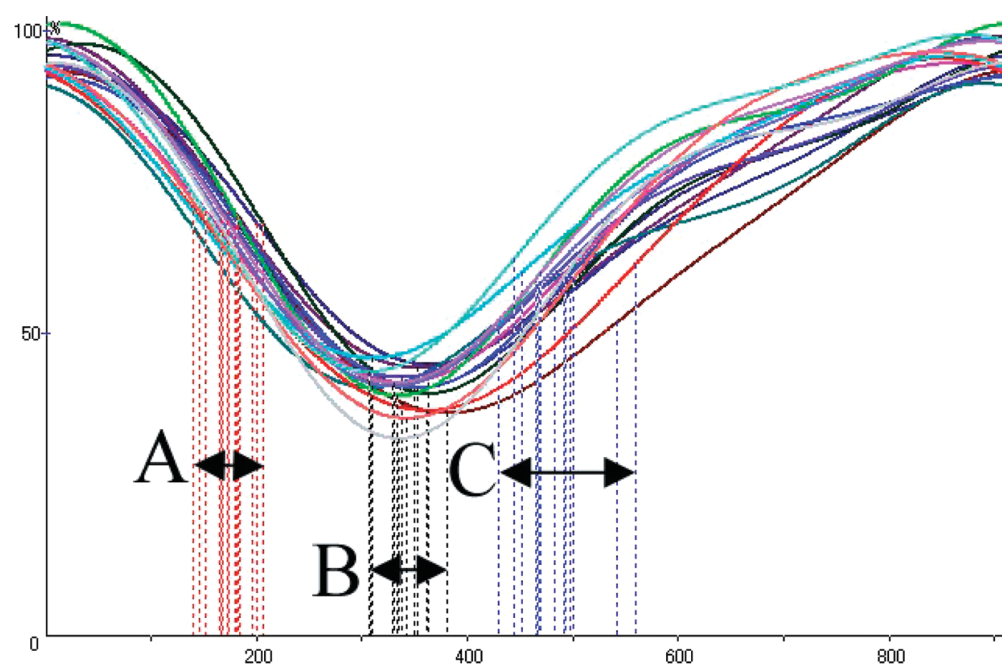

Fig 3. Regional left ventricular TVCs obtained by "cardioGRAF" at rest. The dashed lines demonstrate the TPE, TES and TPF for each of the 17 segments. Arrows show the range from the earliest timing to the latest one in each phase (MD). (A) MD-TPE, (B) MDTES, (C) MD-TPF2. FDC, first derivative curve; MD, maximal difference; TES, time to end systole (ms); TPE, time to peak ejection (ms); TPF, time to peak filling (ms); TVC, time-volume curve. 


\section{Age and Heart Rate}

No significant differences were confirmed among the 4 groups for age or heart rate $(\mathrm{G}-\mathrm{C}=57.9 \pm 12.6$ years, $\mathrm{G}-1=$ $63.3 \pm 13.4$ years, $\mathrm{G}-2=67.5 \pm 11.1$ years, and $\mathrm{G}-3=66.3 \pm 14.1$ years; $\mathrm{G}-\mathrm{C}=65.9 \pm 10.6$ beats $/ \mathrm{min}, \mathrm{G}-1=70.2 \pm 13.3$ beats $/ \mathrm{min}$, G- $2=70.3 \pm 13.5$ beats $/ \mathrm{min}$, and G-3 $=71.1 \pm 11.0$ beats $/ \mathrm{min}$ ), as shown in Table 1.

\section{Global LV Functional Indices}

Table 2 shows the global LV functional indices. The EF was significantly greater in G-C subjects than in G-2 and G-3 patients; it was significantly greater in G-1 patients than in G-2 and G-3 patients and in G-2 patients than in G-3 patients. However, there was no significant difference between G-C
Table 2 Values for Global LV Functions

\begin{tabular}{lcccc}
\hline \hline & $G-C$ & $G-1$ & $G-2$ & $G-3$ \\
\hline$E F(\%)$ & $68.0 \pm 7.1$ & $60.0 \pm 10.0$ & $47.6 \pm 16.7$ & $33.7 \pm 14.6$ \\
$P E R E D V / s$ & $3.2 \pm 0.4$ & $2.9 \pm 0.6$ & $2.2 \pm 0.7$ & $1.5 \pm 0.6$ \\
$P F R E D V / s$ & $2.4 \pm 0.4$ & $2.1 \pm 0.5$ & $1.7 \pm 0.6$ & $1.3 \pm 0.4$ \\
$1 / 3 F R E D V / s$ & $2.1 \pm 0.5$ & $1.5 \pm 0.4$ & $1.3 \pm 0.6$ & $0.9 \pm 0.4$ \\
\hline
\end{tabular}

Data are expressed as means $\pm S D$.

$L V$, left ventricular; EF, ejection fraction; PER, peak ejection rate; EDV, end-diastolic volume; $1 / 3 F R$, first-third filling rate.
A

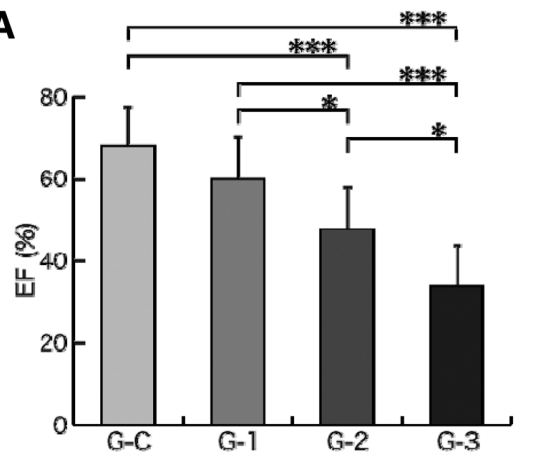

C
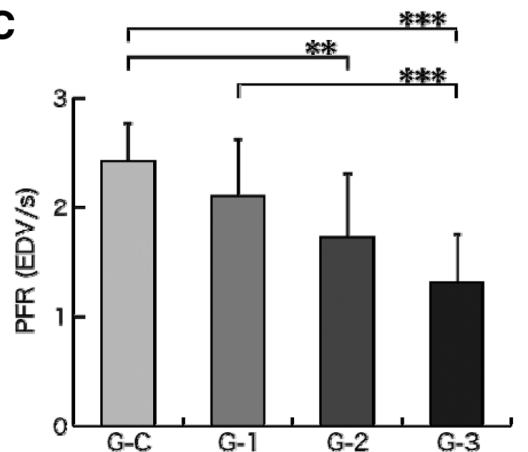

B

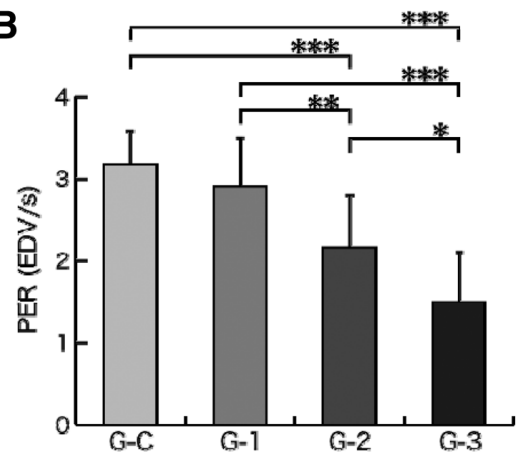

D

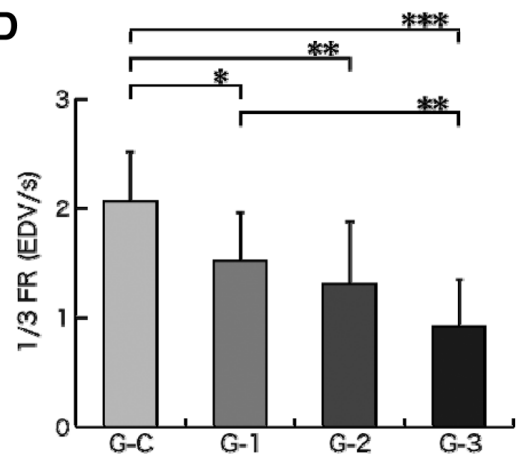

Fig 4. Comparison of global LV function among the 4 groups. EF, ejection fraction; PER, peak ejection rate; PFR, peak filling rate, 1/3FR, first third filling rate. ${ }^{*} \mathrm{p}<0.05, * * \mathrm{p}<$ $0.01, * * * \mathrm{p}<0.001$.
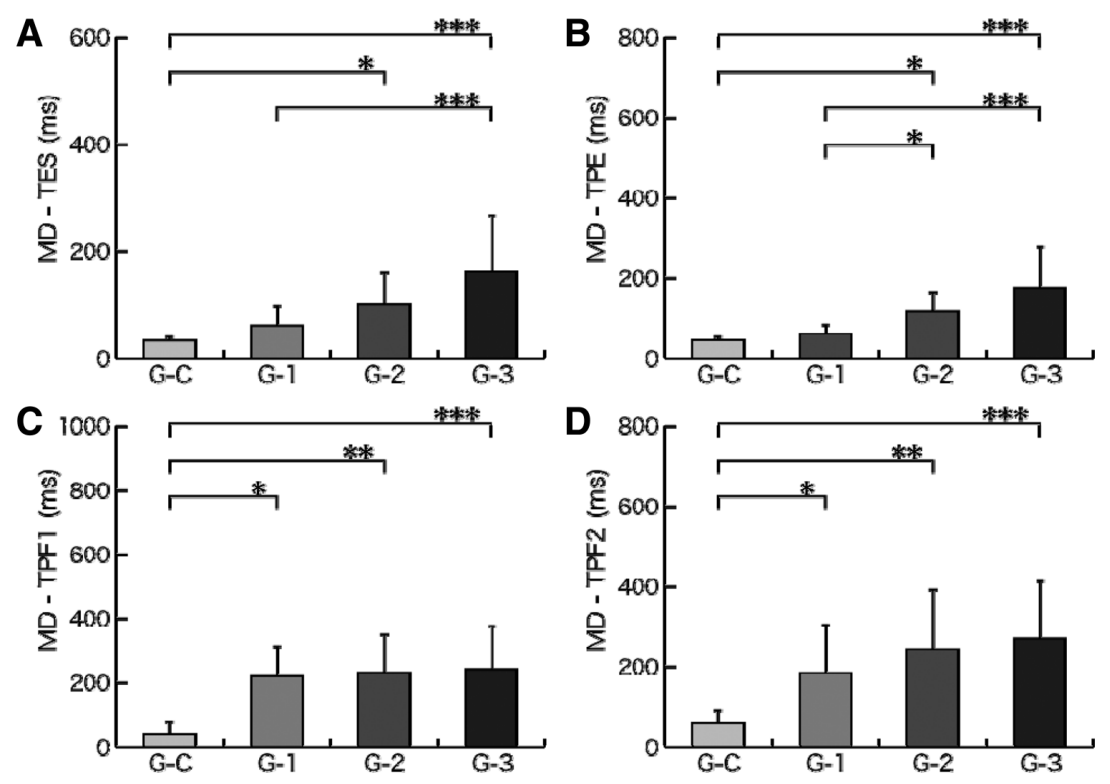

Fig 5. Comparison of maximal difference (MD) among the 4 groups. TES, time to end systole; TPE, time to peak ejection; TPF1, time from end systole to peak filling; TPF2, time from 0 to peak filling. ${ }^{*} \mathrm{p}<0.05,{ }^{* *} \mathrm{p}<0.01$, $* * * \mathrm{p}<0.001$ 
A

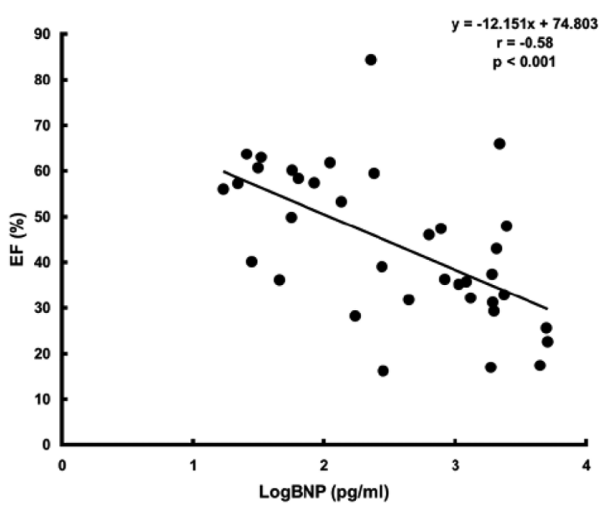

C

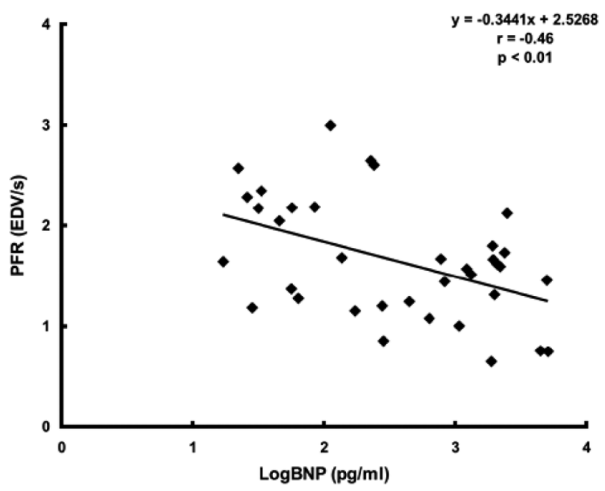

B

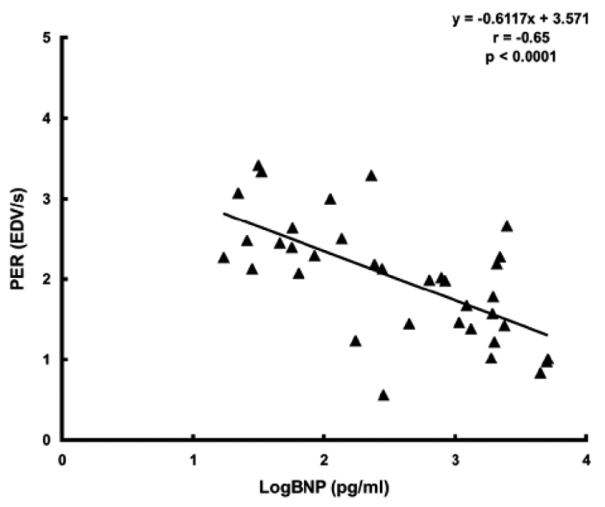

D

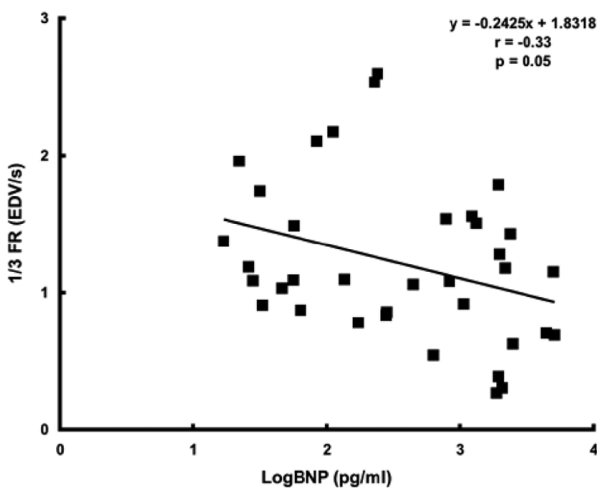

Fig 6. Correlation between plasma B-type natriuretic peptide (BNP) level and global LV function using "cardioGRAF". Scatter plots of LogBNP and EF, PER, PFR and 1/3FR are shown. EF, PER and PFR demonstrate a significant negative correlation with LogBNP. See Figs 1-5 for abbreviations.

subjects and G-1 patients (G-C=68.0 $\pm 7.1 \%, \mathrm{G}-1=60.0 \pm$ $10.0 \%$, G-2 $=47.6 \pm 16.7 \%$, and G-3 $=33.7 \pm 14.6 \%$, Fig $4 \mathrm{~A}$ ). The PER values showed the same pattern of differences as LVEF (G-C $=3.2 \pm 0.4 \mathrm{EDV} / \mathrm{s}, \mathrm{G}-1=2.9 \pm 0.6 \mathrm{EDV} / \mathrm{s}, \mathrm{G}-2=2.2 \pm$ $0.7 \mathrm{EDV} / \mathrm{s}$ and $\mathrm{G}-3=1.5 \pm 0.6 \mathrm{EDV} / \mathrm{s}$, Fig 4B). PFR was significantly greater in G-C subjects than in G-2 and G-3 patients, and in G-1 patients than in G-3 patients $(\mathrm{G}-\mathrm{C}=2.4 \pm$ $0.4 \mathrm{EDV} / \mathrm{s}, \mathrm{G}-1=2.1 \pm 0.5 \mathrm{EDV} / \mathrm{s}, \mathrm{G}-2=1.7 \pm 0.6 \mathrm{EDV} / \mathrm{s}$ and G-3=1.3 $\pm 0.4 \mathrm{EDV} / \mathrm{s}$, Fig $4 \mathrm{C}$ ). Only $1 / 3 \mathrm{FR}$ was significantly greater in G-C subjects than in the other groups, and in G-1 patients than in $\mathrm{G}-3$ patients $(\mathrm{G}-\mathrm{C}=2.1 \pm 0.5 \mathrm{EDV} / \mathrm{s}, \mathrm{G}-1=1.5 \pm$ $0.4 \mathrm{EDV} / \mathrm{s}, \mathrm{G}-2=1.3 \pm 0.6 \mathrm{EDV} / \mathrm{s}$ and $\mathrm{G}-3=0.9 \pm 0.4 \mathrm{EDV} / \mathrm{s}$, Fig 4D).

\section{Systolic and Diastolic Synchronous Indices}

MD-TES was significantly smaller in G-C subjects than in G-2 and G-3 patients, and in G-1 patients than in G-3 patients $(\mathrm{G}-\mathrm{C}=32.9 \pm 7.2 \mathrm{~ms}, \mathrm{G}-1=60.4 \pm 38.2 \mathrm{~ms}, \mathrm{G}-2=101.1 \pm$ $57.9 \mathrm{~ms}$, and G-3=161.5 $\pm 102.4 \mathrm{~ms}$, Fig 5A). MD-TPE was significantly smaller in both G-C and G-1 individuals than in G-2 and G-3 patients. However, neither MD-TES nor MD-TPE was significantly different between G-C and G-1, or $\mathrm{G}-2$ and $\mathrm{G}-3(\mathrm{G}-\mathrm{C}=44.9 \pm 11.2 \mathrm{~ms}, \mathrm{G}-1=60.7 \pm 22.4 \mathrm{~ms}$, G-2=115.1 $\pm 46.8 \mathrm{~ms}$, and G-3=173.6 $\pm 103.5 \mathrm{~ms}$, Fig 5B). MD-TPF1 was significantly smaller in G-C subjects than in the other groups $(\mathrm{G}-\mathrm{C}=39.2 \pm 37.5 \mathrm{~ms}, \mathrm{G}-1=219.7 \pm 93.3 \mathrm{~ms}$, G-2 $=230.8 \pm 117.2 \mathrm{~ms}$ and G-3 $=241.2 \pm 135.3 \mathrm{~ms}$, Fig 5C). MD-TPF2 was also significantly smaller in G-C subjects than in the other groups $(\mathrm{G}-\mathrm{C}=58.2 \pm 34.1 \mathrm{~ms}, \mathrm{G}-1=183.9 \pm$ $119.5 \mathrm{~ms}, \mathrm{G}-2=243.4 \pm 146.3 \mathrm{~ms}$ and $\mathrm{G}-3=269.6 \pm 143.5 \mathrm{~ms}$, Fig 5D).
Correlation Between Plasma BNP Level and LV Function and Dyssynchrony

There were statistically significant negative correlations between the plasma BNP level and LVEF $(\mathrm{r}=-0.55, \mathrm{p}<1 \times$ $\left.10^{-3}\right)$, PER $\left(r=-0.58, \mathrm{p}<5 \times 10^{-4}\right)$ and PFR $(r=-0.41, \mathrm{p}<0.02)$ (Figs 6A-C), and a nonsignificant negative correlation between plasma BNP levels and 1/3FR $(r=-0.34, p=0.05)$ (Fig 6D). There were statistically significant positive correlations between plasma BNP level and MD-TES, MD-TPE $(r=0.37, p<0.05, r=0.50, p<0.01)$ (Figs 7A,B) and not significant with MD-TPF1, MD-TPF2 ( $\mathrm{r}=-0.0004, \mathrm{p}=0.99, \mathrm{r}=0.20$, $\mathrm{p}=0.24)($ Figs $7 \mathrm{C}, \mathrm{D})$.

\section{Discussion}

The principal finding of the present study is the presence of LV diastolic dyssynchrony even in the early stage of HF. This feature was amply demonstrated by the significant differences between the G-C subjects and G-1 patients in MD-TPF1 and MD-TPF2 (Figs 5C,D). In addition, we demonstrated that the plasma BNP level was significantly correlated with not only LVEF, PER and PFR, but also with MD-TES and MD-TPE (Figs 4A-C,5A,B). Interestingly, there were significant differences between the G-C subjects and G-1 patients in the 1/3FR, MD-TPF1 and MD-TPF2 values, which did not correlate with the plasma BNP level.

\section{Functional Parameters}

To assess LV systolic function, we used the LVEF and PER data obtained by cardioGRAF. We have previously validated these parameters by comparing them with the results of equilibrium radionuclide angiography (ERNA) and 

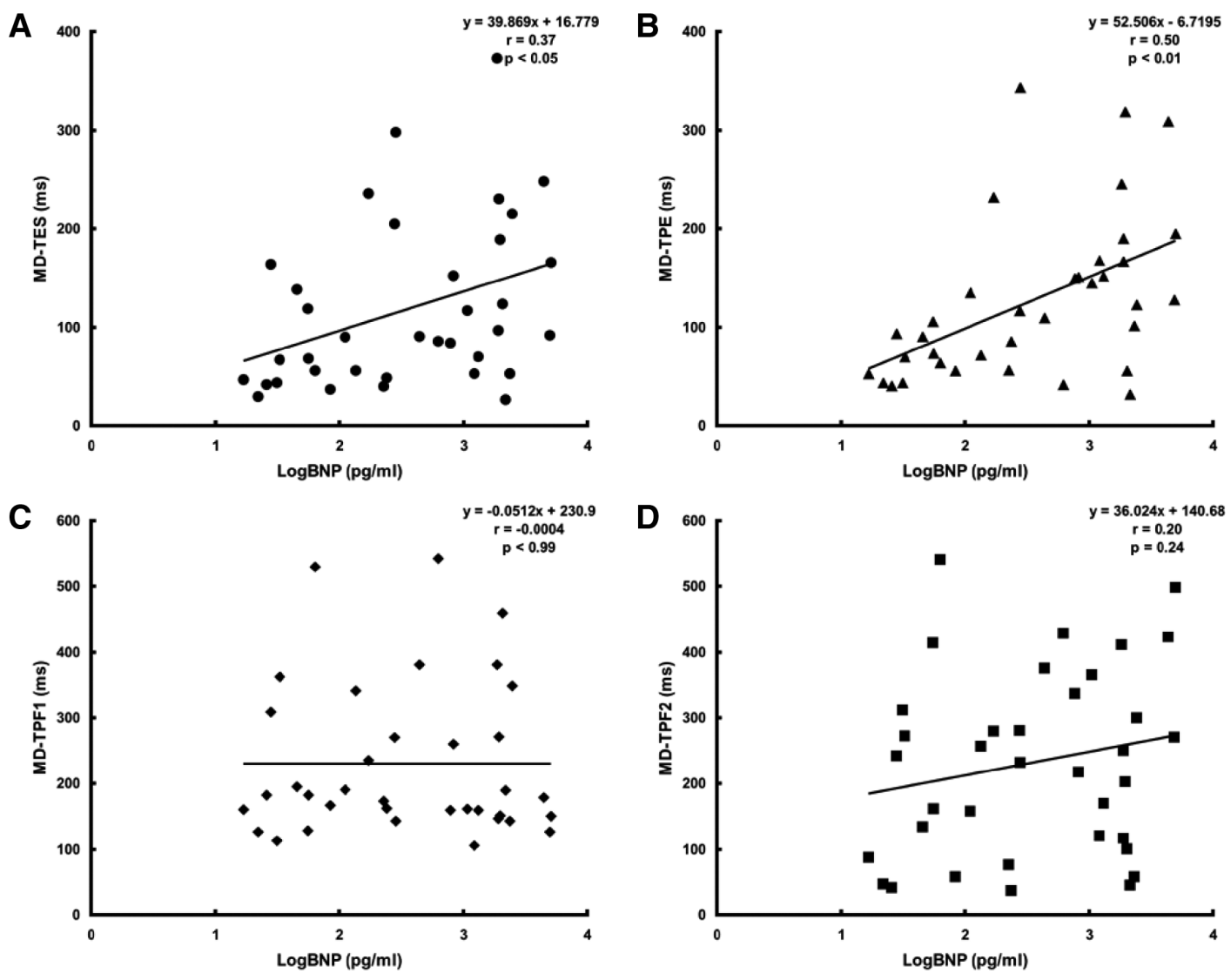

Fig 7. Correlation between plasma BNP levels and MD using "cardioGRAF". Scatter plots of LogBNP and MD-TES, MD-TPE, MD-TPF1 and MD-TPF2 are shown. There is a significant positive correlation between $\operatorname{LogBNP}$ and the systolic parameters MD-TES and MD-TPE. See Figs 1-6 for abbreviations.

2-dimensional echocardiography! ${ }^{10}$ In the present study the values for LVEF and PER decreased in the same order as the NYHA classification (Figs 4A,B), which was the anticipated result and consistent with previous reports! ${ }^{15,16}$ To assess LV diastolic function, we selected PFR and 1/3FR. PFR is used as a conventional standard of diastolic function because it reflects the rate of LV relaxation. Myocardial relaxation involves a complex interaction of both active and passive processes, such as intrinsic relaxation properties and loading conditions. Usually, PFR is defined as the maximum FR during the whole diastolic period in GMPS studies, but in the present study, PFR was the maximum FR during whole diastole and was not been limited to the peak FR during early diastole. During early isovolumic diastole, myocardial relaxation is an active, energy-dependent process and the 1/3FR enables evaluation of early diastolic performance! 7 It should be noted that in this study there was a significant difference between the G-C subjects and G-1 patients in 1/3FR, but not in PFR (Figs 4D,C), which suggests that, at least, there was a significant difference in early diastolic function between normal subjects and asymptomatic HF patients.

\section{Systolic and Diastolic Dyssynchrony}

It has already been proven that LV dyssynchrony adversely influences cardiac function in the advanced stage of $\mathrm{HF}$ caused by myocardial infarction, cardiomyopathy and valvular disease, among other causes ${ }^{18}$ In those patients, cardiac resynchronization therapy has been recognized as improving not only LVEF and exercise tolerance, but also morbidity and mortality 19,20 However, it has been unclear if LV dyssynchrony is present during the early stage of HF, so echocardiography, ERNA and phase analysis using GMPS have been used to assess LV dyssynchrony?21,22 Suffoletto et al reported a novel analysis for LV dyssynchrony using speckle tracking imaging (STI) by echocardiography, but because it frequently produces artifacts and requires highquality images there are doubts about the reproducibility and optimal frame rate of this method 23

We have developed a new program that focuses on the evaluation of regional LV systolic/diastolic indices using GMPS. We have validated the systolic/diastolic temporal indices obtained by cardioGRAF by comparing them with those obtained by ERNA and STI ${ }^{24}$ With regard to the systolic synchronous indices, MD-TES and MD-TPE in the G-C subjects were not different from those obtained from G-1 patients (Figs 6A,B). By contrast, the diastolic synchronous indices, MD-TPF1 and MD-TPF2 were significantly smaller in G-C subjects than in G-1 patients (Figs 6C,D). These results suggest that there are significant differences between normal subjects and asymptomatic HF patients in diastolic dyssynchrony, but not in systolic dyssynchrony. Sugihara et al reported that asynchrony of myocardial contraction was inversely correlated with the parameters of LV systolic/diastolic functions. ${ }^{25}$ In the present study, we investigated the relationship between LV dyssynchrony and plasma BNP level, which is related to LV systolic/diastolic function. 


\section{Relationship With Plasma BNP Level}

Previous human studies have suggested correlations between plasma BNP level and LV systolic/diastolic function parameters such as LVEF, end-diastolic pressure and pulmonary capillary wedge pressure (PCWP) ${ }^{26-28}$ In this study, we demonstrated that the plasma BNP level significantly correlated with not only systolic/diastolic functional parameters (Figs 6A-C), but also those of systolic dyssynchrony (Figs 7A,B). To date the relationship between plasma BNP level and systolic dyssynchrony has been unclear. Wang et al demonstrated by echocardiography that systolic dyssynchrony might reflect LV wall stress in HF patients, and moreover, Iwanaga et al reported that LV wall stress accurately accounts for the increase in plasma BNP level in HF patients! ${ }^{16}$ Therefore, we speculated that LV wall stress that increased the plasma BNP level might produce systolic dyssynchrony in HF patients, but we could not clarify the detailed mechanism underlying such a relationship. In the present study the plasma BNP level showed significant negative correlations with LVEF, PER and PFR (Figs 6AC), but not with 1/3FR (Fig 6D). In addition, the plasma BNP level did not correlate with indices of diastolic dyssynchrony, such as MD-TPF1 and MD-TPF2 (Figs 7C,D).

Although it has been the mainstream practise to distinguish between DHF and SHF, the process underlying the development of HF has been unclear. There have been various arguments suggesting that diastolic dysfunction may develop in advance of systolic dysfunction or in parallel with the process of HF ${ }^{29-31}$ Wang et al recently reported that, in DHF, diastolic dyssynchrony was significantly correlated with parameters of diastolic dysfunction, such as relaxation time constant tau and mean PCWP6

Here we have demonstrated that diastolic dyssynchrony might appear even in the early stage of HF and is not correlated with plasma BNP level, whereas systolic dyssynchrony might affect the plasma BNP level. Thus, at least in clinical practice, our novel cardioGRAF indices would be helpful for assessing the development of HF.

\section{Study Limitations}

In this study, we assessed our patients without distinguishing between ischemic and nonischemic diseases, because of the small sample size. Further study with a distinction between these forms of disease, in a greater number of patients, is necessary. Furthermore, in general, LV dyssynchrony involves interventricular dyssynchrony between the left and right ventricles and intraventricular dyssynchrony occurs between LV walls. In the present study we could only detect intraventricular dyssynchrony using cardioGRAF, suggesting that this is now a limitation of GMPS.

\section{Conclusion}

Diastolic dyssynchrony was demonstrated even in the early stage of HF and did not correlate with the plasma BNP level; however, systolic dyssynchrony might affect it.

\section{Acknowledgments}

We thank Teruo Takahashi, Hiroshi Akimoto, and Akira Yakuwa for their technical assistance with the GMPS study, and Daiichi Radioisotope Laboratories/FUJIFILM RI Pharma, Co Ltd for cooperation in development of cardioGRAF.

\section{References}

1. Senni M, Tribouilloy CM, Rodeheffer RJ, Jacobsen SJ, Evans JM,
Baily KR, et al. Congestive heart failure in the community: A study of all incident cases in Olmsted County, Minnesota, in 1991. Circulation 1998; 98: 2282-2289.

2. Vasan RS, Larson MG, Benjamin EJ, Evans JC, Reiss CK, Levy D. Congestive heart failure in subjects with normal versus reduced left ventricular ejection fraction. J Am Coll Cardiol 1999; 33: 1948-1955.

3. Redfield MM, Jacobsen SJ, Burnet JC, Mahoney DW, Bailey KR, Rodeheffer RJ. Burden of systolic and diastolic ventricular dysfunction in the community: Appreciating the scope of the heart failure epidemic. JAMA 2003; 289: 194-202.

4. Matsuzaki M, Sasayama S, Aizawa $\mathrm{Y}$, Imaizumi T, Kato H, Kawaguchi $\mathrm{H}$, et al. Guideline for the diagnosis and treatment of chronic heart failure. Circ J 2000; 64(Suppl IV): 1023-1079.

5. Xiao HB, Brecker SJ, Gibson DG. Effects of abnormal activation on the time course of the left ventricular pressure pulse in dilated cardiomyopathy. Br Heart J 1992; 68: 403-407.

6. Wang J, Kurrelmeyer KM, Torre-Amione G, Nagueh SF. Systolic and diastolic dyssynchrony in patients with diastolic heart failure and the effect of medical therapy. J Am Coll Cardiol 2007; 49: 88-96.

7. Germano G, Kiat H, Kavanagh PB, Moriel M, Mazzanti M, Su HT, et al. Automatic quantification of ejection fraction from gated myocardial perfusion SPECT. J Nucl Med 1995; 36: 2138-2147.

8. Nakata T, Katagiri Y, Odawara Y, Eguchi M, Kuroda M, Tsuchihashi $\mathrm{K}$, et al. Two- and three-dimensional assessment of myocardial perfusion and function by using technetium-99m sestamibi gated SPECT with a combination of count and image-based techniques. $J$ Nucl Cardiol 2000; 7: 623-632.

9. Kumita S, Cho K, Nakajo H, Toba M, Uwamori M, Mizumura S, et al. Assessment of left ventricular diastolic function with electrocardiography-gated myocardial perfusion SPECT: Comparison with multigated equilibrium radionuclide angiography. J Nucl Cardiol 2001; 8: $568-574$.

10. Yamamoto A, Hosoya T, Takahashi N, Iwahara S, Munakata K. Quantification of left ventricular regional functions using ECG-gated myocardioal perfusion SPECT: Validation of left ventricular systolic functions. Ann Nucl Med 2006; 7: 449-466.

11. Lubien E, DeMaria A, Krishnaswamy P, Clopton P, Koon J, Kazanegra $\mathrm{R}$, et al. Utility of B-natriuretic peptide in detecting diastolic dysfunction: Comparison with Doppler velocity recordings. Circulation 2002; 105: $595-601$.

12. Maisel AS, McCord J, Nowak RM, Hollander JE, Wu AH, Duc P, et al. Bedside B-Type natriuretic peptide in the emergency diagnosis of heart failure with reduced or preserved ejection fraction: Results from the Breathing Not Properly multinational study. J Am Coll Cardiol 2003; 41: 2010-2017.

13. Mckee PA, Castelli WP, McNamara PM, Kannel WP. The natural history of congestive heart failure: The Framingham study. $N$ Engl $J$ Med 1971; 285: $1441-1446$

14. American Heart Association Working group. Standardized myocardial segmentation and nomenclature for tomographic imaging of the heart: A statement for healthcare professionals from the Cardiac Imaging Committee of the Council Cardiology of the American Heart Association. Circulation 2000; 105: 539-542.

15. Nakae I, Matsuo S, Koh T, Mitsunami K, Horie M. Left ventricular systolic/diastolic function evaluated by quantitative ECG-gated SPECT: Comparison with echocardiography and plasma BNP analysis. Ann Nucl Med 2005; 19: 447-454.

16. Iwanaga Y, Nishi I, Furuichi S, Noguchi T, Sase K, Kihara Y, et al. B-type natriuretic peptide strongly reflects diastolic wall stress in patients with chronic heart failure: Comparison between systolic and diastolic heart failure. J Am Coll Cardiol 2006; 47: 742-748.

17. Grossman W. Diastolic dysfunction in congestive heart failure. $N$ Engl J Med 1991; 325: 1557-1564.

18. Farwell D, Patel NR, Hall A, Ralph S, Sulke AN. How many people with heart failure are appropriate for biventricular resynchronization? Eur Heart J 2000; 21: 1246-1250.

19. Abraham WT, Fisher WG, Smith AL, Delurgio DB, Leon AR, Loh $\mathrm{E}$, et al. Cardiac resynchronization in chronic heart failure. $N$ Engl $J$ Med 2002; 346: $1845-1853$.

20. Cleland JG, Daubert JC, Erdmann E, Freemantle N, Gras D, Kappenberger L, et al. The effect of cardiac resynchronization on morbidity and mortality in heart failure. N Engl J Med 2005; 352: $1594-1597$.

21. Somsen GA, Verberne HJ, Burri H, Ratib O, Righeti A. Ventricular mechanical dyssynchrony and resynchronization therapy in heart failure: A new indication for Fourier analysis of gated blood-pool radionuclide ventriculography. Nucl Med Commun 2006; 27:105-112.

22. Chen J, Garcia EV, Folks RD, Cooke CD, Faber TL, Tauxe EL, et al. Onset of left ventricular mechanical contraction as determined by phase analysis of ECG-gated myocardial perfusion SPECT imaging: 
Development of a diagnostic tool for assessment of cardiac mechanical dyssynchrony. J Nucl Cardiol 2005; 12: 687-695.

23. Suffoletto MS, Dohi K, Cannesson M, Saba S, Gorcsan J III. Novel spackle-tracking radial strain from routine black and white echocardiographic images to quantify dyssynchrony and predict response to cardiac resynchronization therapy. Circulation 2006; 113: 960-968.

24. Yamamoto A, Takahashi N, Munakata K, Hosoya T, Shiiba M, Okuyama T, et al. Global and regional evaluation of systolic and diastolic left ventricular temporal parameters using a novel program for ECG-gated myocardial perfusion SPECT: Validation by comparison with gated equilibrium radionuclide angiography and speckle-tracking radial strain from echocardiography. Ann Nucl Med 2007; 21: $115-121$.

25. Sugihara H, Yonekura Y, Matsumoto T, Sasaki Y. Relationship between asynchronous myocardial contraction and left ventricular systolic and diastolic function: Assessment using the ECG-Gated polar map with ${ }^{99 m}$ Tc-methoxy-isobutyl isonitrile. Circ J 2005; 69: 183-187.

26. Maeda K, Tsutamoto T, Wada A, Hisanaga T, Kinoshita M. Plasma brain natriuretic peptide as a biochemical markaer of high left ventricular end-diastolic pressure in patients with symptomatic left ventricular dysfunction. Am Heart J 1998; 135: 825-832.
27. Troughton RW, Prior DL, Pereira JJ, Martin M, Fogarty A, Morehead A, et al. Plasma B-type natriuretic peptide levels in systolic heart failure: Importance of left ventricular diastolic function and right ventricular systolic function. J Am Coll Cardiol 2004; 43: 416-422.

28. Yamamoto K, Burnett JC, Jougasaki M, Nishimura RA, Bailey KR, Saito Y, et al. Superiority of brain natriuretic peptide as a hormonal marker of ventricular systolic and diastolic dysfunction and ventricular hypertrophy. Hypertension 1996; 28: 988-994.

29. Kitabatake A, Inoue M, Asao M, Tanouchi J, Masuyama T, Abe H, et al. Transmitral blood flow reflecting diastolic behavior of the left ventricle in health and disease. Circ J 1982; 46: 92-102.

30. Bountioukos M, Schinkel A, Bax JJ, Lampropoulos S, Poldermans D. The impact of hypertension on systolic and diastolic left ventricular function: A tissue Doppler echocardiographic study. Am Heart J 2006; 151: 1323. e7-e12.

31. Melenovsky V, Borlaug BA, Rosen B, Hay I, Ferruci L, Morell CH, et al. Cardiovascular features of heart failure with preserved ejection fraction versus nonfailing hypertensive left ventricular hypertrophy in the urban Baltimore community: The role of atrial remodeling/dysfunction. J Am Coll Cardiol 2007; 49: 198-207. 